\title{
Impact of ethnicity, unemployment and economy on mental disorders : A study from Western Nepal
}

Banerjee $\mathrm{I}^{1}$, Banerjee $\mathrm{I}^{2}$, Roy $\mathrm{R}^{3}$, Sathian $\mathrm{B}^{4}$, Khadka $\mathrm{S}^{5}$, Chakraborty $\mathrm{PK}^{6}$, Saha A7 , Pugazhandhi B ${ }^{8}$

${ }^{1}$ Lecturer, Department of Pharmacology, Manipal College of Medical Sciences, Pokhara, Nepal.

2 Post Doctorate Trainee, M.Ch Urology, SMS Medical College, India

${ }^{3}$ Assistant Professor, Department of Physiology, Manipal College of Medical Sciences, Pokhara, Nepal.

${ }^{4}$ Assistant Professor, Department of Community Medicine, Manipal College of Medical Sciences, Pokhara, Nepal.

${ }^{5}$ Lecturer, English, Kalika Multiple Campus, Pokhara, Nepal.

6 Professor of Psychiatry, Hospital Director, Manipal Teaching Hospital, Pokhara, Nepal.

${ }^{7}$ Professor and Head of the Department of Pharmacology, Manipal College of Medical Sciences, Pokhara.

${ }^{8}$ Lecturer, Department of Anatomy, Manipal College of Medical Sciences, Nepal

\section{Chief Editor}

Dr. Padam Prasad Simkhada

Technical Editor

Dr. Nishida Chandrasekharan

Formatting Editor

Dr. Ram Lakhan

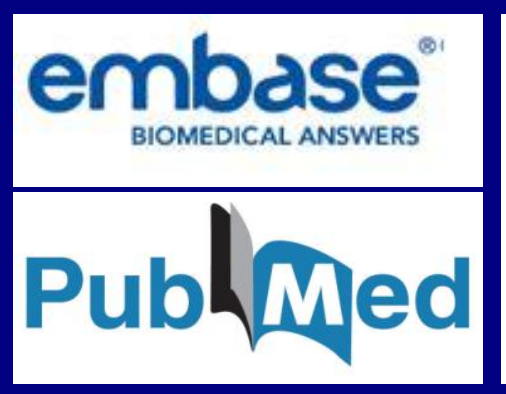

This title

is indexed

in SciVerse

Scopus

Improving research

results through

analytical power

\section{Original Article}

\section{Corresponding Author}

Dr. Indrajit Banerjee MD

Lecturer, Department of Pharmacology,

Manipal College of Medical Sciences,

Deep Heights- 16

Pokhara, Nepal

E-mail: indrajit18@gmail.com
Background

The causes of mental health problems and ethnic variation are poorly understood. The main objective of the study was to find out about psychiatric diseases which frequently occur in Western Nepal for which hospitalization is required. The specific objective was to research about the 
economic condition of the psychiatric patients and the prevalence of common psychiatric disorder witnessed among hospitalized patients who belong to diverse ethnic and cultural groups in Western Nepal.

\section{Materials and Methods}

It was a cross sectional study which was conducted in between $1^{\text {st }}$ October 2009 and 31th March 2010 between at Manipal teaching hospital, Pokhara, Nepal. Odds ratios and adjusted odds ratio and their 95\% confidence intervals (95\% $\mathrm{Cl})$ were calculated. $\mathrm{p}<0.05$ was considered as statistically significant.

\section{Results}

Out of 240 cases the commonest cases of psychiatric disorders include Schizophrenia, Schizotypal and Delusion Disorders 36.3\%, Mood Disorders 27.9\%, Neurotic, stressrelated and somatoform disorders $15.8 \%$. Study based on ethnicity revealed that the majority of patients were Dalit [ $n=72$ ] followed by Brahmin [ $n=66]$, Chettri [ $n=46]$, Newar [ $n=19]$, Gurung [ $n=17]$, others [ $=13$ ] and Magar and Pun [ $=7$ ]. Most of the patients were <40yrs [n=191] unemployed [ $n=199]$, monthly family income $<10000$ $\mathrm{NPR} /$ month [ $\mathrm{n}=187]$, students $[\mathrm{n}=102]$ housewives [ $\mathrm{n}=$ 74,] job holders [ $n=17]$. Study showed that Mental and Behavioural disorder due to Psychoactive Substance abuse, Schizophrenia, Schizotypal and Delusion Disorders Mood (Affective) Disorders, Neurotic, stress-related and somatoform disorders were prevalent among unemployed patients [OR $8.170(\mathrm{Cl} 1.062,62.853)]$, [OR $3.033(\mathrm{Cl} 1.334$, 6.897)], [OR $0.413(\mathrm{Cl} 0.199,0.856)[\mathrm{OR} \quad 0.228(\mathrm{Cl} 0.089$, $0.583)$ ]as compared to employed patients $(p=0.001)$.

\section{Conclusion}

Schizophrenia was the commonest psychiatric disorder among the low socio-economic class of like Dalits. The study showed that culture based differences concerning mental health is further mediated by poverty, unemployment and dearth of family income which leads to high prevalence of psychiatric illness among Nepalese population. Based on the finding of the study, interventions should target these factors to minimise the load of various psychiatric illness among poor Dalit Nepalese population.

Keywords: Culture, Ethnicity, Nepal, Psychiatry

\section{Introduction}

The causes of mental health problems and ethnic variation are poorly understood ${ }^{1}$. From Income point of view, Nepal is a poor developing country which is situated in South East Asia $^{2}$. Its population structure includes multi-lingual and multi ethnic diverse population. The main source of income of Nepalese people is agriculture. Most parts of Nepal are occupied by mountainous and hills where people don't have good access to health care facilities. Many health related researches that have been undertaken in Nepal are mainly about the prevalence of mental illnesses. A study carried out in Janakpur, Eastern Nepal reports that the commonest forms of disorder is schizophrenia $30 \%$ followed by bipolar disorder $25 \%{ }^{3}$. Another research finding indicates that Schizophrenia $50.1 \%$ was the commonest psychiatric disorder followed by mood disorders $33.5 \%$ and substance use disorder $9.3 \%{ }^{4}$ whereas study conducted by Risal confirms that the commonest psychiatric disorder were anxiety disorder as seen in a primary care setting followed by depressive and unexplained somatic symptoms ${ }^{5}$. Nepalese population consists of a wide range of ethnic/tribal groups namely the Brahmin, the Chettri, the Gurung, the Newar, the Puns, the Magar, the Dalit ${ }^{6}$. The data related to ethnic variation and corresponding mental illness is lacking in western region in particular and Nepal in general. The main objective of the study was to research about the commonest psychiatric disease which occurs in Western Nepal for which hospitalization is required. The specific objective of the study was to research about the economic condition of the psychiatric patients and to identify prevalence of common psychiatric disorder among hospitalized patients in different ethnic/cultural groups.

\section{Material and Methods}

\section{Study design and the participants:}

This research involves was a cross sectional study which was conducted at Manipal teaching hospital, Pokhara, Nepal, a tertiary care hospital situated in Western Nepal.

\section{Data collection:}

The data was collected between 1st October 2009 and 31th March 2010 at Psychiatric ward in Manipal Teaching hospital. The information and data collected about different psychiatric disorders were Organic including symptomatic mental disorder F00-F9, Mental and Behavioural disorder due to Psychoactive Substance abuse F10-19, Schizophrenia, Schizotypal and Delusion Disorders F20-29, Mood (Affective) Disorders F30-39, Neurotic, stress-related and somatoform disorders F40-48, Behavioural syndromes associated with physiological disturbances and physical factors F50-F59 and Mental retardation F70-F79. The collected data include socio-demographic details such as age (<40yearsand>40years), gender (male and female), occupation (Shopkeeper, Farmer, Labour, Retired, Jobholder, Housewife, Student and Others), housewife, religion (Muslim, Christian, Buddhist, Hindu), ethnicity (Magar, Pun, Gurung, Newar, Dalit, Chettri, Brahmin and Others), employment (employed and unemployed), monthly income NPR ( $<10000 /$ month and $>10000 /$ month).

\section{Inclusion criteria:}

A total number of 240 cases of critical nature with all types of Psychiatric disorders were included in the study. The diagnosis of the disease was based on ICD-10 (Tenth revision) Classification of mental and Behavioural disorders; Diagnostic Criteria for Research ${ }^{7}$. The total number of cases 
Ethnicity, unemployment, economy and mental disorders Result:

includes psychiatric inpatients and those who were both inpatient and outpatients.

\section{Exclusion criteria:}

All the outpatients were excluded as the research aims to study about the psychiatric patients who were critically ill for which hospitalization is required.

\section{Sample size calculation:}

For $95 \%$ confidence interval and, significance level $\alpha=5 \%$, $\mathrm{P}=70 \%, \mathrm{Q}=30 \%$, allowable error $=10 \%$ of $\mathrm{P}$, required sample size was 165 . Prior to the study a pilot study was done in 50 patients admitted in the psychiatric inpatients and it was found that $70 \%$ of the patients were having monthly income $<10000$ NPR/month ${ }^{8}$.

\section{Outcome Variable:}

The main outcome variable was the commonest disorder seen among the psychiatric inpatients.

\section{Explanatory variables:}

The Socio demographic and psychiatric disorders have been defined at individual level. Factors which were taken into consideration at individual level were Age $(<40$ years and $>40$ years), gender (male and female), monthly income ( $<10000 /$ month and $>10000 /$ month), employment of the patient (employed and unemployed), occupation (housewife, laborer, student, farmer, retired and others), religion (Hindu, Buddhist, Muslim), ethnicity (Brahmin, Chettri, Newar, Dalit and others). The causes of mental health problems and ethnic variation are poorly understood.

\section{Ethical committee approval:}

The Research was conducted in accordance to latest version of the Declaration of Helsinki. Prior the study, ethical committee approval was taken from the institutional ethical committee, Manipal Teaching hospital, Pokhara, Nepal.

\section{Data management and statistical analysis:}

The data collected was analyzed using Excel 2003, R 2.8.0 Statistical Package for the Social Sciences (SPSS) for Windows Version 16.0 (SPSS Inc; Chicago, IL, USA) and EPI Info 3.5.1 Windows Version. Chi square test was used to observe the difference between different variables and strength of the relationship with logistic regression. $p<0.05$ was considered as statistically significant. We calculated odds ratios and adjusted odds ratio) and their 95\% confidence intervals $(95 \% \mathrm{Cl}) . \mathrm{p}<0.05$ was considered as statistically significant ${ }^{9}$.

\section{Prevalence of Psychiatric disorders}

The research finding revealed that common forms of psychiatric disorders were Schizophrenia, Schizotypal and Delusion Disorders 36.3\%, Mood (Affective) Disorders 27.9\%, Neurotic, stress-related and somatoform disorders $15.8 \%$, Mental and behavioural disorder due to Psychoactive Substance abuse 11.3\%, Behavioural syndromes associated with physiological disturbances and physical factors $4.2 \%$, Organic including symptomatic mental disorders $2.9 \%$ and Mental retardation $1.7 \%$ respectively. The majority of psychiatric patients were <40yrs (191), 49 patients were >40yrs. Among 191 patients who were $<40$ years, the common psychiatric disorders were Schizophrenia, Schizotypal and Delusion Disorders(35.6\%) followed by Mood (Affective) Disorders 25.1\%, Neurotic, stress-related and somatoform disorders $17.8 \%$, Mental and behavioural disorder due to Psychoactive Substance abuse $12.6 \%$ respectively. Among the patients $>40 \mathrm{yrs}$, most common disorders were Schizophrenia, Schizotypal and Delusion Disorders and Mood (Affective) Disorders (38.8\%). Out of 110 female patients, most of them suffered from Mood (Affective) Disorders 30.9\%, Schizophrenia, Schizotypal and Delusion Disorders $27.3 \%$ followed by Neurotic, stress-related and somatoform disorders $24.5 \%$ respectively. Out of total figure, 130 patients were concerned Schizophrenia, Schizotypal and Delusion Disorders $43.8 \%$ was the commonest followed by Mood (Affective) Disorders $25.4 \%$ respectively $(P=0.002)$. Most of the patients were unemployed $(n=199)$ and among them $39.2 \%$ suffered from Schizophrenia, Schizotypal and Delusion Disorders followed by Mood (Affective) Disorders $25.1 \%$ and Neurotic, stress-related and somatoform disorders $13.6 \%$ respectively. Among the employed patients $(n=41)$ most of the patients had been suffering from mood and affective disorders $41.5 \%$ followed by Neurotic, stressrelated and somatoform disorders and Schizophrenia, Schizotypal and Delusion Disorders $26.8 \%$ and $22 \%$ respectively $(p=0.02)$. Most of the patients had monthly income <10000 per month $(n=187)$. They were suffering from Schizophrenia, Schizotypal and Delusion Disorders ( $n=67)$, mood affective disorders Neurotic, stress-related and somatoform disorders $(n=33)$. Religion wise, the majority of the patients were Hindus $(a n=200)$ followed by Buddhist $(n=25)$, Christian ( $n=9)$ and Muslim $(n=6)$. Among Hindus, Schizophrenia, Schizotypal and Delusion Disorders were common types of psychiatric disorders followed by mood affective disorders $28.5 \%$, Neurotic, stress-related and somatoform disorders $18.5 \%$ Mental and behavioural disorder primarily caused due to Psychoactive Substance abuse $11 \%$ respectively. On the basis of ethinic backround, most of the patients were Dalit $[n=72]$ followed by Brahmin $(n=66)$, Chettri $(n=46)$, Newar $(n=19)$, Gurung $(n=170)$, others $(n=13)$ and Magar and Pun $(n=7)$. 
Table 1: Socio economic, demographic details and psychiatric disorder

\begin{tabular}{|c|c|c|c|c|c|c|c|c|}
\hline \multicolumn{2}{|c|}{$\begin{array}{c}\text { Socio Economic and demographic } \\
\text { Factors }\end{array}$} & $\begin{array}{l}\text { Organic including } \\
\text { symptomatic } \\
\text { mental disorder } \\
\text { F00-F9 }\end{array}$ & $\begin{array}{c}\text { Mental and } \\
\text { behavioral } \\
\text { disorder due to } \\
\text { Psychoactive } \\
\text { Substance abuse } \\
\text { F10-19 }\end{array}$ & $\begin{array}{c}\text { Schizophrenia, } \\
\text { Schizotypal and } \\
\text { Delusion } \\
\text { Disorders F20- } \\
29\end{array}$ & $\begin{array}{c}\text { Mood } \\
\text { (Affective) } \\
\text { Disorders F 30- } \\
39\end{array}$ & $\begin{array}{l}\text { Neurotic, stress- } \\
\text { related and } \\
\text { somatoform } \\
\text { disorders F40- } \\
48\end{array}$ & $\begin{array}{c}\text { Behavioral } \\
\text { syndromes } \\
\text { associated with } \\
\text { physiological } \\
\text { disturbances and } \\
\text { physical factors } \\
\text { F50-F59 }\end{array}$ & \multirow[t]{4}{*}{$\begin{array}{l}\text { Mental } \\
\text { retardation } \\
\text { F70-F79 }\end{array}$} \\
\hline \multirow{3}{*}{ Age } & $>40 y r s[49]$ & $\begin{array}{c}3(6.1) \\
{[1.3,16.9]}\end{array}$ & $\begin{array}{c}3(6.1) \\
{[1.3,16.9]}\end{array}$ & $\begin{array}{c}19(38.8) \\
{[25.2,53.8]}\end{array}$ & $\begin{array}{c}19(38.8) \\
{[25.2,53.8]}\end{array}$ & $\begin{array}{c}4(8.2) \\
{[2.3,29.6]}\end{array}$ & $\begin{array}{c}1(2) \\
{[0.1,10.9]}\end{array}$ & \\
\hline & $<40 y r s[191]$ & $\begin{array}{c}4(2.1) \\
{[0.6,5.3]}\end{array}$ & $\begin{array}{c}24(12.6) \\
{[8.2,18.1]}\end{array}$ & $\begin{array}{c}68(35.6) \\
{[28.8,42.8]}\end{array}$ & $\begin{array}{c}48(25.1) \\
{[19.1,31.9]}\end{array}$ & $\begin{array}{c}34(17.8) \\
{[12.7,24]}\end{array}$ & $\begin{array}{c}9(4.7) \\
{[2.2,8.8]}\end{array}$ & \\
\hline & P Value & \multicolumn{6}{|c|}{$0.113 x$} & \\
\hline \multirow{3}{*}{ Gender } & Female[110] & $\begin{array}{l}4(3.6) \\
{[1,9]}\end{array}$ & $\begin{array}{c}7(6.4) \\
{[2.6,12.7]}\end{array}$ & $\begin{array}{c}30(27.3) \\
{[19.2,36.6]}\end{array}$ & $\begin{array}{c}34(30.9) \\
{[22.4,40.4]}\end{array}$ & $\begin{array}{c}27(24.5) \\
{[16.8,33.7]}\end{array}$ & $\begin{array}{c}5(4.5) \\
{[1.5,10.3]}\end{array}$ & $\begin{array}{c}3(2.7) \\
{[0.6,7.8]}\end{array}$ \\
\hline & Male[130] & $\begin{array}{c}3(2.3) \\
{[0.5,6.6]}\end{array}$ & $\begin{array}{c}20(15.4) \\
{[9.7,22.8]}\end{array}$ & $\begin{array}{c}57(43.8) \\
{[35.2,52.8]}\end{array}$ & $\begin{array}{c}33(25.4) \\
{[18.2,33.8]}\end{array}$ & $\begin{array}{c}11(8.5) \\
{[4.3,14.6]}\end{array}$ & $\begin{array}{c}5(3.8) \\
{[1.3,8.7]}\end{array}$ & $\begin{array}{c}1(0.8) \\
{[0,4.2]}\end{array}$ \\
\hline & P Value & \multicolumn{7}{|c|}{$0.002+$} \\
\hline \multirow{3}{*}{ Employment } & Employed[41] & $\begin{array}{c}2(4.9) \\
{[0.6,16.5]}\end{array}$ & $\begin{array}{c}1(2.4) \\
{[0.1,12.9]}\end{array}$ & $\begin{array}{c}9(22) \\
{[10.6,37.6]}\end{array}$ & $\begin{array}{c}17(41.5) \\
{[26.3,57.9]}\end{array}$ & $\begin{array}{c}11(26.8) \\
{[14.2,42.9]}\end{array}$ & $\begin{array}{c}1(2.4) \\
{[0.1,12.9]}\end{array}$ & $\begin{array}{c}0(0) \\
{[0,8.6]}\end{array}$ \\
\hline & $\begin{array}{c}\text { Unemployed } \\
\text { [199] }\end{array}$ & $\begin{array}{c}5(2.5) \\
{[0.8,5.8]}\end{array}$ & $\begin{array}{c}26(13.1) \\
{[8.7,18.6]}\end{array}$ & $\begin{array}{c}78(39.2) \\
{[32.4,46.3]}\end{array}$ & $\begin{array}{c}50(25.1) \\
{[19.3,31.7]}\end{array}$ & $\begin{array}{c}27(13.6) \\
{[9.1,19.1]}\end{array}$ & $\begin{array}{c}9(4.5) \\
{[2.1,8.4]}\end{array}$ & $\begin{array}{c}4(2) \\
{[0.6,5.1]}\end{array}$ \\
\hline & P Value & \multicolumn{7}{|c|}{$0.02+$} \\
\hline \multirow{8}{*}{$\begin{array}{l}\text { Monthly } \\
\text { Income }\end{array}$} & $\begin{array}{c}>10000 / \text { month } \\
{[53]}\end{array}$ & $\begin{array}{c}2(3.8) \\
{[0.5,13]}\end{array}$ & $\begin{array}{c}8(15.1) \\
{[6.7,27.6]}\end{array}$ & $\begin{array}{c}20(37.7) \\
{[24.8,52.1]}\end{array}$ & $\begin{array}{c}17(32.1) \\
{[19.9,46.3]}\end{array}$ & $\begin{array}{c}5(9.4) \\
{[3.1,20.7]}\end{array}$ & $\begin{array}{c}1(1.9) \\
{[0,10.1]}\end{array}$ & $\begin{array}{c}0(0) \\
{[0,6.7]}\end{array}$ \\
\hline & $\begin{array}{c}<10000 / \text { month } \\
{[187]}\end{array}$ & $\begin{array}{c}5(2.7) \\
{[0.9,6.1]}\end{array}$ & $\begin{array}{c}19(10.2) \\
{[6.2,15.4]}\end{array}$ & $\begin{array}{c}67(35.8) \\
{[29,43.2]}\end{array}$ & $\begin{array}{c}50(26.7) \\
{[20.5,33.7]}\end{array}$ & $\begin{array}{c}33(17.6) \\
{[12.5,23.9]}\end{array}$ & $\begin{array}{c}9(4.8) \\
{[2.2,8.9]}\end{array}$ & $\begin{array}{c}4(2.1) \\
{[0.6,5.4]}\end{array}$ \\
\hline & P Value & \multicolumn{7}{|c|}{$0.510 x$} \\
\hline & Muslim[6] & $\begin{array}{c}0(0) \\
{[0,45.9]}\end{array}$ & $\begin{array}{c}0(0) \\
{[0,45.9]}\end{array}$ & $\begin{array}{c}3(50) \\
{[11.8,88.2]}\end{array}$ & $\begin{array}{c}2(33.3) \\
{[4.3,77.7]}\end{array}$ & $\begin{array}{c}0(0) \\
{[0,45.9]}\end{array}$ & $\begin{array}{c}0(0) \\
{[0,45.9]}\end{array}$ & $\begin{array}{c}1(16.7) \\
{[0.4,64.1]}\end{array}$ \\
\hline & Christian[9] & $\begin{array}{c}0(0) \\
{[0,33.6]}\end{array}$ & $\begin{array}{c}3(33.3) \\
{[7.5,70.1]}\end{array}$ & $\begin{array}{c}4(44.4) \\
{[13.7,78.8]}\end{array}$ & $\begin{array}{c}0(0) \\
{[0,33.6]}\end{array}$ & $\begin{array}{c}1(11.1) \\
{[0.3,48.2]}\end{array}$ & $\begin{array}{c}1(11.1) \\
{[0.3,48.2]}\end{array}$ & $\begin{array}{c}0(0) \\
{[0,33.6]}\end{array}$ \\
\hline & Buddhist[25] & $\begin{array}{c}0(0) \\
{[0,13.7]}\end{array}$ & $\begin{array}{c}2(8) \\
{[1,26]}\end{array}$ & $\begin{array}{c}14(56) \\
{[34.9,75.6]}\end{array}$ & $\begin{array}{c}8(32) \\
{[14.9,53.5]}\end{array}$ & $\begin{array}{c}0(0) \\
{[0,13.7]}\end{array}$ & $\begin{array}{c}0(0) \\
{[0,13.7]}\end{array}$ & $\begin{array}{c}1(4) \\
{[0.1,20.4]}\end{array}$ \\
\hline & Hindu[200] & $\begin{array}{c}7(3.5) \\
{[1.4,7.1]}\end{array}$ & $\begin{array}{l}22(11) \\
{[7,16.2]}\end{array}$ & $\begin{array}{c}66(33) \\
{[26.5,40]}\end{array}$ & $\begin{array}{c}57(28.5) \\
{[22.4,35.3]}\end{array}$ & $\begin{array}{c}37(18.5) \\
{[13.4,24.6]}\end{array}$ & $\begin{array}{c}9(4.5) \\
{[2.1,8.4]}\end{array}$ & $\begin{array}{c}2(1) \\
{[0.1,3.6]}\end{array}$ \\
\hline & P Value & & & & $0.113 x$ & & & \\
\hline \multirow{8}{*}{ Ethnicity } & $\begin{array}{c}\text { Magar, Pun } \\
\text { [7] }\end{array}$ & $\begin{array}{c}0(0) \\
{[0,41]}\end{array}$ & $\begin{array}{c}0(0) \\
{[0,41]}\end{array}$ & $\begin{array}{c}5(71.4) \\
{[29,96.3]}\end{array}$ & $\begin{array}{c}2(28.6) \\
{[3.7,71]}\end{array}$ & $\begin{array}{c}0(0) \\
{[0,41]}\end{array}$ & $\begin{array}{c}0(0) \\
{[0,41]}\end{array}$ & $\begin{array}{c}0(0) \\
{[0,41]}\end{array}$ \\
\hline & $\begin{array}{c}\text { Others } \\
\text { [13] }\end{array}$ & $\begin{array}{c}0(0) \\
{[0,24.7]}\end{array}$ & $\begin{array}{c}2(15.4) \\
{[1.9,45.4]}\end{array}$ & $\begin{array}{c}6(46.2) \\
{[19.2,74.9]}\end{array}$ & $\begin{array}{c}3(23.1) \\
{[5,53.8]}\end{array}$ & $\begin{array}{c}1(7.7) \\
{[0.2,36]}\end{array}$ & $\begin{array}{c}0(0) \\
{[0,24.7]}\end{array}$ & $\begin{array}{c}1(7.7) \\
{[0.2,36]}\end{array}$ \\
\hline & $\begin{array}{c}\text { Gurung } \\
\lceil 17]\end{array}$ & $\begin{array}{c}0(0) \\
{[0,19.5]}\end{array}$ & $\begin{array}{c}2(11.8) \\
{[1.5,36.4]}\end{array}$ & $\begin{array}{c}9(52.9) \\
{[27.8,77]}\end{array}$ & $\begin{array}{c}5(29.4) \\
{[10.3,56]}\end{array}$ & $\begin{array}{c}0(0) \\
{[0,19.5]}\end{array}$ & $\begin{array}{c}0(0) \\
{[0,19.5]}\end{array}$ & $\begin{array}{c}1(5.9) \\
{[0.1,28.7]}\end{array}$ \\
\hline & $\begin{array}{c}\text { Newar } \\
{[19]}\end{array}$ & $\begin{array}{c}0(0) \\
{[0,17.6]}\end{array}$ & $\begin{array}{c}2(10.5) \\
{[1.3,33.1]}\end{array}$ & $\begin{array}{c}7(36.8) \\
{[16.3,61.6]}\end{array}$ & $\begin{array}{c}8(42.1) \\
{[20.3,66.5]}\end{array}$ & $\begin{array}{c}2(10.5) \\
{[1.3,33.1]}\end{array}$ & $\begin{array}{c}0(0) \\
{[0,17.6]}\end{array}$ & $\begin{array}{c}0(0) \\
{[0,17.6]}\end{array}$ \\
\hline & $\begin{array}{l}\text { Dalit } \\
\text { [72] }\end{array}$ & $\begin{array}{c}5(6.9) \\
{[2.3,15.5]}\end{array}$ & $\begin{array}{l}15(20.8) \\
{[12.2,32]}\end{array}$ & $\begin{array}{c}22(30.6) \\
{[20.2,42.5]}\end{array}$ & $\begin{array}{c}13(18.1) \\
{[10,28.9]}\end{array}$ & $\begin{array}{c}9(12.5) \\
{[5.9,22.4]}\end{array}$ & $\begin{array}{c}8(11.1) \\
{[4.9,20.7]}\end{array}$ & $\begin{array}{c}0(0) \\
{[0,5]}\end{array}$ \\
\hline & $\begin{array}{c}\text { Chettri } \\
\text { [46] }\end{array}$ & $\begin{array}{c}0(0) \\
{[0,7.7]}\end{array}$ & $\begin{array}{c}0(0) \\
{[0,7.7]}\end{array}$ & $\begin{array}{c}21(45.7) \\
{[30.9,61.0]}\end{array}$ & $\begin{array}{c}13(28.3) \\
{[16,43.5]}\end{array}$ & $\begin{array}{c}12(26.1) \\
{[14.3,41.1]}\end{array}$ & $\begin{array}{c}0(0) \\
{[0,7.7]}\end{array}$ & $\begin{array}{c}0(0) \\
{[0,7.7]}\end{array}$ \\
\hline & Brahmin & $\begin{array}{c}2(3) \\
{[0.4,10.5]}\end{array}$ & $\begin{array}{c}6(9.1) \\
\lceil 3.4,18.7\rceil\end{array}$ & $\begin{array}{c}17(25.8) \\
{[15.8,38]}\end{array}$ & $\begin{array}{c}23(34.8) \\
{[23.5,47.6]}\end{array}$ & $\begin{array}{c}14(21.2) \\
{[12.1,33]}\end{array}$ & $\begin{array}{c}2(3) \\
{[0.4,10.5]}\end{array}$ & $\begin{array}{c}2(3) \\
{[0.4,10.5]}\end{array}$ \\
\hline & P Value & & & & $0.004+$ & & & \\
\hline \multirow{9}{*}{ Occupation } & $\begin{array}{c}\text { Shopkeeper } \\
{[4]}\end{array}$ & $\begin{array}{c}0(0) \\
{[0,60.2]}\end{array}$ & $\begin{array}{c}1(25) \\
{[0.6,80.6]}\end{array}$ & $\begin{array}{c}0(0) \\
{[0,60.2]}\end{array}$ & $\begin{array}{c}2(50) \\
{[6.8,93.2]}\end{array}$ & $\begin{array}{c}1(25) \\
{[0.6,80.6]}\end{array}$ & $\begin{array}{c}0(0) \\
{[0,60.2]}\end{array}$ & $\begin{array}{c}0(0) \\
{[0,60.2]}\end{array}$ \\
\hline & $\begin{array}{l}\text { Others } \\
\text { [7] }\end{array}$ & $\begin{array}{c}0(0) \\
{[0,41]}\end{array}$ & $\begin{array}{c}0(0) \\
{[0,41]}\end{array}$ & $\begin{array}{c}2(28.6) \\
{[3.7,71]}\end{array}$ & $\begin{array}{c}1(14.3) \\
{[0.4,57.9]}\end{array}$ & $\begin{array}{c}0(0) \\
{[0,41]}\end{array}$ & $\begin{array}{c}0(0) \\
{[0,41]}\end{array}$ & $\begin{array}{c}4(57.1) \\
{[18.4,19.1]}\end{array}$ \\
\hline & $\begin{array}{c}\text { Farmer } \\
\text { [9] }\end{array}$ & $\begin{array}{c}1(11.1) \\
{[0.3,48.2]}\end{array}$ & $\begin{array}{c}0(0) \\
{[0,33.6]}\end{array}$ & $\begin{array}{c}4(44) \\
{[13.7,78.8]}\end{array}$ & $\begin{array}{c}2(22.2) \\
\lceil 2.8,60]\end{array}$ & $\begin{array}{c}2(22.2) \\
\lceil 2.8,60]\end{array}$ & $\begin{array}{c}0(0) \\
{[0,33.61}\end{array}$ & $\begin{array}{c}0(0) \\
{[0,33.6]}\end{array}$ \\
\hline & $\begin{array}{c}\text { Labour } \\
\text { [11] }\end{array}$ & $\begin{array}{c}1(9.1) \\
{[0.2,41.3]}\end{array}$ & $\begin{array}{c}0(0) \\
{[0,28.5]}\end{array}$ & $\begin{array}{c}2(18.2) \\
{[2.3,51.8]}\end{array}$ & $\begin{array}{c}6(54.5) \\
{[23.4,83.4]}\end{array}$ & $\begin{array}{c}2(18.2) \\
{[2.3,51.8]}\end{array}$ & $\begin{array}{c}0(0) \\
{[0,28.5]}\end{array}$ & $\begin{array}{c}0(0) \\
{[0,28.5]}\end{array}$ \\
\hline & $\begin{array}{c}\text { Retired } \\
{[16]}\end{array}$ & $\begin{array}{c}3(18.8) \\
{[4,45.6]}\end{array}$ & $\begin{array}{c}1(6.3) \\
{[0.2,30.2]}\end{array}$ & $\begin{array}{c}5(31.3) \\
{[11,58.7]}\end{array}$ & $\begin{array}{c}6(37.5) \\
{[15.2,64.6]}\end{array}$ & $\begin{array}{c}1(6.3) \\
{[0.2,30.2]}\end{array}$ & $\begin{array}{c}0(0) \\
{[0,20.6]}\end{array}$ & $\begin{array}{c}0(0) \\
{[0,20.6]}\end{array}$ \\
\hline & $\begin{array}{c}\text { Jobholder } \\
\text { [17] }\end{array}$ & $\begin{array}{c}0(0) \\
{[0,19.5]}\end{array}$ & $\begin{array}{c}0(0) \\
{[0,19.5]}\end{array}$ & $\begin{array}{c}3(17.6) \\
{[3.8,43.4]}\end{array}$ & $\begin{array}{c}7(41.2) \\
{[18.4,67.1]}\end{array}$ & $\begin{array}{c}6(35.3) \\
{[14.2,61.7]}\end{array}$ & $\begin{array}{c}1(5.9) \\
{[0.1,28.7]}\end{array}$ & $\begin{array}{c}0(0) \\
{[0,19.5]}\end{array}$ \\
\hline & $\begin{array}{c}\text { Housewife } \\
{[74]}\end{array}$ & $\begin{array}{c}2(2.7) \\
{[0.3,9.4]}\end{array}$ & $\begin{array}{c}3(4.1) \\
{[0.8,11.4]}\end{array}$ & $\begin{array}{c}23(31.1) \\
{[20.8,42.9]}\end{array}$ & $\begin{array}{c}23(31) \\
{[20.8,42.9]}\end{array}$ & $\begin{array}{c}18(24.3) \\
{[15.1,35.7]}\end{array}$ & $\begin{array}{c}5(6.8) \\
{[2.2,15.1]}\end{array}$ & $\begin{array}{c}0(0) \\
{[0,4.9]}\end{array}$ \\
\hline & $\begin{array}{c}\text { Student } \\
\text { [102] }\end{array}$ & $\begin{array}{c}0(0) \\
{[0,3.6]}\end{array}$ & $\begin{array}{c}22(21.6) \\
{[14,30.8]}\end{array}$ & $\begin{array}{c}48(47.1) \\
{[37.1,57.2]}\end{array}$ & $\begin{array}{c}20(19.6) \\
{[12.4,28.6]}\end{array}$ & $\begin{array}{c}8(7.8) \\
{[3.4,14.9]}\end{array}$ & $\begin{array}{c}4(3.9) \\
{[1.1,9.7]}\end{array}$ & $\begin{array}{c}0(0) \\
{[0,3.6]}\end{array}$ \\
\hline & P Value & \multicolumn{7}{|c|}{$0.000+$} \\
\hline
\end{tabular}

$+p<0.05$, statistically significant., $\times p>0.05$, statistically not significant. 
Ethnicity, unemployment, economy and mental disorders

Table 2: Logistic Regression Table of Socio Economic, Demographic factors and various Psychiatric Disorders

\begin{tabular}{|c|c|c|c|c|c|c|c|c|}
\hline \multirow{2}{*}{\multicolumn{2}{|c|}{$\begin{array}{c}\text { Socio Economic and } \\
\text { demographic } \\
\text { Factors }\end{array}$}} & $\begin{array}{c}\text { Organic including } \\
\text { symptomatic } \\
\text { mental disorder } \\
\text { F00-F9 }\end{array}$ & $\begin{array}{c}\text { Mental and } \\
\text { behavioral } \\
\text { disorder due to } \\
\text { Psychoactive } \\
\text { Substance abuse } \\
\text { F10-19 }\end{array}$ & $\begin{array}{c}\text { Schizophrenia, } \\
\text { Schizotypal and } \\
\text { Delusion Disorders } \\
\text { F20-29 }\end{array}$ & $\begin{array}{l}\text { Mood (Affective) } \\
\text { Disorders F 30-39 }\end{array}$ & $\begin{array}{l}\text { Neurotic, stress- } \\
\text { related and } \\
\text { somatoform } \\
\text { disorders F40-48 }\end{array}$ & $\begin{array}{c}\text { Behavioral } \\
\text { syndromes } \\
\text { associated with } \\
\text { physiological } \\
\text { disturbances and } \\
\text { physical factors } \\
\text { F50-F59 }\end{array}$ & $\begin{array}{l}\text { Mental } \\
\text { retardation } \\
\text { F70-F79 }\end{array}$ \\
\hline & & $\begin{array}{c}\text { Odds Ratio } \\
\text { (Confidence } \\
\text { Interval) }\end{array}$ & $\begin{array}{l}\text { Odds Ratio } \\
\text { (Confidence } \\
\text { Interval) }\end{array}$ & $\begin{array}{c}\text { Odds Ratio } \\
\text { (Confidence } \\
\text { Interval) }\end{array}$ & $\begin{array}{l}\text { Odds Ratio } \\
\text { (Confidence } \\
\text { Interval) }\end{array}$ & $\begin{array}{l}\text { Odds Ratio } \\
\text { (Confidence } \\
\text { Interval) }\end{array}$ & $\begin{array}{l}\text { Odds Ratio } \\
\text { (Confidence } \\
\text { Interval) }\end{array}$ & $\begin{array}{c}\text { Odds Ratio } \\
\text { (Confidence } \\
\text { Interval) }\end{array}$ \\
\hline \multirow{2}{*}{ Age } & $>40 y r s$ & 1 & 1 & 1 & 1 & 1 & 1 & 1 \\
\hline & $<40 y r s$ & $\begin{array}{c}0.328(0.071,1.517) \\
x\end{array}$ & $\begin{array}{c}2.204(0.635 \\
7.644) x\end{array}$ & $0.873(0.457,1.666) x$ & $\begin{array}{c}\text { 1.887(0.974, } \\
3.655) \mathrm{x}\end{array}$ & $\begin{array}{c}2.436(0.821,7.230) \\
x\end{array}$ & $\begin{array}{c}2.374(0.293 \\
19.197) \mathrm{x}\end{array}$ & - \\
\hline \multirow[b]{2}{*}{ Gender } & Female & 1 & 1 & 1 & 1 & 1 & 1 & 1 \\
\hline & Male & $\begin{array}{c}0.626(0.137,2.859) \\
x\end{array}$ & $\begin{array}{c}2.675(1.086 \\
6.591)^{*}\end{array}$ & $2.082(1.208,3.588)^{\dagger}$ & $\begin{array}{c}0.70(0.432,1.338) \\
x\end{array}$ & $\begin{array}{c}0.284(0.134 \\
0.605)^{\dagger}\end{array}$ & $0.84(0.237,2.981) x$ & $\begin{array}{c}0.276(0.028 \\
2.697) \mathrm{x}\end{array}$ \\
\hline \multirow{2}{*}{$\begin{array}{l}\text { Employ } \\
\text { ment }\end{array}$} & Employed & 1 & 1 & 1 & 1 & 1 & 1 & 1 \\
\hline & Unemployed & $\begin{array}{c}0.503(0.094,2.685) \\
x\end{array}$ & $\begin{array}{c}6.012(0.792 \\
45.621) x\end{array}$ & $2.292(1.038,5.062)^{*}$ & $\begin{array}{c}0.474(0.235 \\
0.953)^{*}\end{array}$ & $\begin{array}{c}0.428(0.192 \\
0.954)^{\dagger}\end{array}$ & $\begin{array}{c}\text { 1.895(0.233, } \\
15.379) x\end{array}$ & - \\
\hline \multirow[b]{2}{*}{$\begin{array}{l}\text { Monthly } \\
\text { Income }\end{array}$} & $>10000 /$ month & 1 & 1 & 1 & 1 & 1 & 1 & 1 \\
\hline & $<10000 /$ month & $\begin{array}{c}0.701(0.132,3.718) \\
x\end{array}$ & $\begin{array}{l}0.636(0.261 \\
1.548) x\end{array}$ & $0.921(0.490,1.731) x$ & $\begin{array}{c}0.773(0.399 \\
1,498) x\end{array}$ & $\begin{array}{c}2.057(0.761,5.563) \\
x\end{array}$ & $\begin{array}{c}2.629(0.326 \\
21.235) x\end{array}$ & - \\
\hline \multirow{4}{*}{ Religion } & Muslim & 1 & 1 & 1 & 1 & 1 & 1 & 1 \\
\hline & Christian & - & - & $0.8(0.101,6.347) x$ & - & - & - & - \\
\hline & Buddhist & - & - & $1.273(0.214,7.581) x$ & $\begin{array}{l}0.941(0.142 \\
6.255) x\end{array}$ & - & - & $\begin{array}{l}0.208(0.011 \\
3.919) x\end{array}$ \\
\hline & Hindu & - & - & $0.493(0.097,2.507) x$ & $\begin{array}{l}0.797(0.142 \\
4.474) x\end{array}$ & - & - & $\begin{array}{l}0.51(0.004 \\
0.653)^{*}\end{array}$ \\
\hline \multirow{7}{*}{ Ethnicity } & Magar, Pun & 1 & 1 & 1 & 1 & 1 & 1 & 1 \\
\hline & Others & - & - & $0.343(0.048,2.457) x$ & $\begin{array}{c}0.750(0.093 \\
6.043) x\end{array}$ & - & - & - \\
\hline & Gurung & - & - & $0.450(0.068,2.998) x$ & $\begin{array}{c}\text { 1.042(0.149, } \\
7.275) \mathrm{x}\end{array}$ & - & - & - \\
\hline & Newar & - & - & $0.233(0.35,1.539) \mathrm{x}$ & $\begin{array}{c}1.818(0.279 \\
11.865) x\end{array}$ & - & - & - \\
\hline & Dalit & - & - & $0.176(0.032,0.978) x$ & $\begin{array}{l}0.551(0.096 \\
3.158) x\end{array}$ & - & - & - \\
\hline & Chettri & - & - & $0.336(0.059,1.913) x$ & $\begin{array}{l}0.985(0.169 \\
5.730) x\end{array}$ & - & - & - \\
\hline & Brahmin & - & - & $0.139(0.025,0.783) x$ & $\begin{array}{l}\text { 1.337(0.240 } \\
7.439) \mathrm{x}\end{array}$ & - & - & - \\
\hline \multirow{8}{*}{$\begin{array}{l}\text { Occupati } \\
\text { on }\end{array}$} & Shopkeeper & 1 & 1 & 1 & 1 & 1 & 1 & 1 \\
\hline & Others & - & - & - & $\begin{array}{l}0.167(0.009 \\
2.984) x\end{array}$ & - & - & - \\
\hline & Farmer & - & - & - & $\begin{array}{l}0.286(0.023 \\
3.523) x\end{array}$ & $\begin{array}{c}0.857(0.055 \\
13.479) x\end{array}$ & - & - \\
\hline & Labour & - & - & - & $\begin{array}{c}1.2(0.121,11.865) \\
x\end{array}$ & $\begin{array}{c}0.667(0.043 \\
10.253) x\end{array}$ & - & - \\
\hline & Retired & - & $0.2(0.01,4.166) x$ & - & $0.6(0.066,5.447) x$ & $\begin{array}{c}0.200(0.010,4.166) \\
x\end{array}$ & - & - \\
\hline & Jobholder & - & - & - & $0.7(0.079,6.224) x$ & $\begin{array}{c}1.636(0.138,19.387) \\
x\end{array}$ & - & - \\
\hline & Housewife & - & $\begin{array}{c}0.127(0.01,1.609) \\
x\end{array}$ & - & $\begin{array}{l}0.451(0.060 \\
3.402) \mathrm{x}\end{array}$ & $\begin{array}{c}0.964(0.094,9.858) \\
x\end{array}$ & - & - \\
\hline & Student & - & $\begin{array}{l}0.825(0.082 \\
8.327) x\end{array}$ & - & $\begin{array}{c}0.244(0.032 \\
1.839) \mathrm{x}\end{array}$ & $\begin{array}{c}0.255(0.024,2.746) \\
x\end{array}$ & 1 & 1 \\
\hline
\end{tabular}

$+p<0.05$, statistically significant. $\times p>0.05$, statistically not significant. 
Among the Dalits, $30.6 \%$ of them had been suffering from Schizophrenia, Schizotypal and Delusion Disorders followed by Mental and behavioural disorder due to Psychoactive substance abuse $20.8 \%$, mood and affective disorders $18.1 \%$, Neurotic, stress-related and somatoform disorders $12.5 \%$, Behavioural syndromes associated with physiological disturbances and physical factors $11.1 \%$, Organic including symptomatic mental disorder $6.9 \%$ respectively.

Psychiatric disorders prevalent among the Brahmins are characterized as Mood (Affective) Disorders 34.8\%, Schizophrenia, Schizotypal and Delusion Disorders 25.8\%, Neurotic, stress-related and somatoform disorders $21.2 \%$, Mental and behavioural disorder resulted owing to Psychoactive Substance abuse 9.1\%, Organic including symptomatic mental disorder $3 \%$, Behavioural syndromes associated with physiological disturbances and physical factors 3\% and Mental retardation 3\% respectively. Among the Chettri, the commonest psychiatric disorder was Schizophrenia, Schizotypal and Delusion Disorders $45.7 \%$ which is followed by Mood (Affective) Disorders 28.3\%, Neurotic, stress-related and somatoform disorders $26.1 \%$ respectively. $(P=0.004)$. The data indicates that most of the patient were students $(n=102)$ followed by housewives $(n=$ 74) job holders $(n=17)$ retired $(n=17)$, labours $(n=11)$, farmers $(n=9)$, others $(n=7)$ and shopkeepers $(n=4)$. Among students the most common psychiatric disorder was Schizophrenia, Schizotypal and Delusion Disorders 47.1\%, mental and behavioural disorder due to Psychoactive Substance abuse 21.6\%, mood (Affective) Disorders 19.6\%, Neurotic, stress-related and somatoform disorders $7.8 \%$, Behavioural syndromes associated with physiological disturbances and physical factors 3.9\% respectively. None of the students were found to be suffering from Organic including symptomatic mental disorder $0 \%$ and mental retardation $0 \%$. Among housewives, the commonest psychiatric disorder was Mood (Affective) Disorders and Schizophrenia, Schizotypal and Delusion Disorders 31\%, followed by Neurotic, stress-related and somatoform disorders 24.3\%, Behavioural syndromes associated with physiological disturbances and physical factors $6.8 \%$, Mental and behavioural disorder because of Psychoactive Substance abuse $4.1 \%$ and Organic including symptomatic mental disorder $2.7 \%$ respectively. None of the housewives were diagnosed to be suffering from Mental retardation $0 \%$. All the values are found to be statistically significant. ( $P$ value $=0.000)($ Table 1$)$

\section{Determinants of socio demographic factors and various} psychiatric disorders by logistic regression

Logistic regression analysis finding shows that the psychiatric disorders like Mental and behavioural disorder due to Psychoactive Substance abuse F10-19, Schizophrenia, Schizotypal and Delusion Disorders F20-29 and Neurotic, stress-related and somatoform disorders F4048 was found more prevalent in males [OR $2.675,95 \%(\mathrm{Cl}$ $1.086,6.591)], \quad[O R 2.082,95 \%(\mathrm{Cl} 1.208,3.588)$ ], [OR $0.284,95 \%(\mathrm{Cl} 0.134,0.605)]$ respectively as compared to female patients. However, in the case of Schizophrenia,
Schizotypal and Delusion Disorders F20-29, it revealed that it was more prevalent among unemployed patients [OR $2.292,95 \%(\mathrm{Cl} 1.038,5.062)]$. All the values were found to be statistically significant (Table 2 ).

Considering the adjusted Odds ratio, it was found that Mental and behavioural disorder due to Psychoactive Substance abuse F10-19, Schizophrenia, Schizotypal and Delusion Disorders F20-29, Neurotic, stress-related and somatoform disorders $\mathrm{F} 40-48$ was [OR3.287,95\%(Cl 1.320, 8.186)], [OR 2.494,95\%(Cl 1.417, 4.391)], [OR 0.191,95\% $(\mathrm{Cl} 0.081,0.449)]$ respectively indicating bigger number of male patients as compared to female ones. Mental and behavioural disorder due to Psychoactive Substance abuse F10-19Schizophrenia, Schizotypal and Delusion Disorders F20-29 Mood(Affective) Disorders F 30-39 Neurotic, stress-related and somatoform disorders $\mathrm{F} 40-48$ was found to be more prevalent in unemployed patients [OR $8.170(\mathrm{Cl} 1.062,62.853)]$, [OR $3.033(\mathrm{Cl} 1.334,6.897)]$, [OR $0.413(\mathrm{Cl} 0.199,0.856)[\mathrm{OR}$ $0.228(\mathrm{Cl} 0.089,0.583)]$ as compared to employed patients. All the values were found to be statistically significant (Table 3).

\section{Table 3: Adjusted Odd Ratio Table}

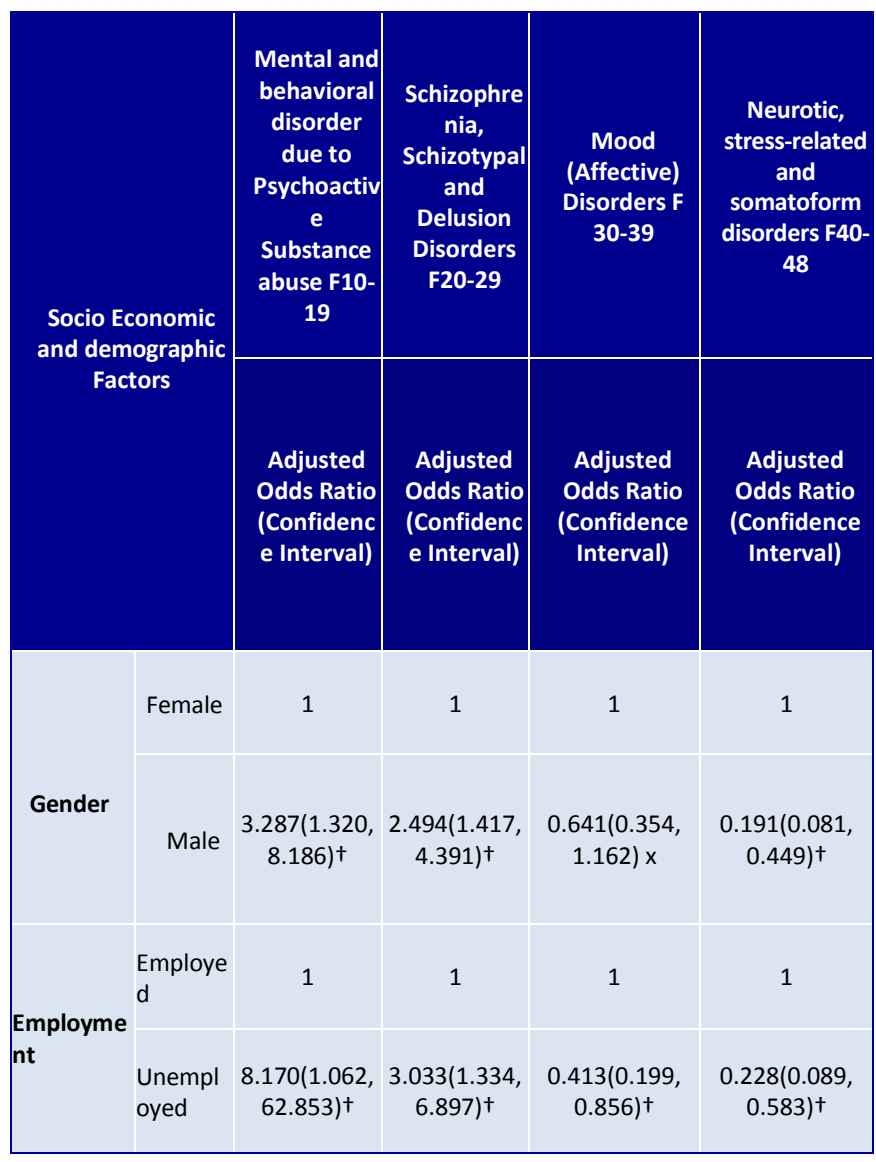

$\dagger p<0.05$, statistically significant.

$\times p>0.05$, statistically not significant. 

research didn't have age limitation of patients, the collected samples moreover belong to the age group $<40 \mathrm{yrs}$. Besides

\section{Prevalence of Psychiatric disorder}

There is a wide variation concerning prevalence of psychiatric illness in Nepal. In this research it was revealed that commonest psychiatric disorder was Schizophrenia, Schizotypal and Delusion Disorders 36.3\% followed by Mood (Affective) Disorders 27.9\%, Neurotic, stress-related and somatoform disorders 15.8\%, Mental and Behavioural disorder due to Psychoactive Substance abuse 11.3\%, Behavioural syndromes associated with physiological disturbances and physical factors $4.2 \%$, Organic including symptomatic mental disorders $2.9 \%$ and Mental retardation $1.7 \%$ respectively. Likewise, the study conducted by Shrestha MR in Kathmandu indicates that common diagnoses were Schizophrenia (50.1\%), mood disorders (33.5\%) and substance use disorder (9.3\%) which corresponds to the finding of this research ${ }^{4}$. However, the finding of this study is contradictory to another research carried out by Risal A et al in Kathmandu Valley, Nepal as his finding concludes that the commonest cause of depression among referred in-patients was anxiety disorder ${ }^{10}$. A study conducted in 2000 at Jiri which is situated in North eastern part of Nepal reported that out of 653 patients, the commonest psychiatric disorder was somatization disorder $11.08 \%$, Generalized anxiety disorder 3.5\%, Depression $3.1 \%$, Mania 3.6\%, Schizophrenia $1.4 \%$ and Antisocial personality disorder $0.2 \%$ which is quite dissimilar to this research finding ${ }^{11}$. A study done by Khattri JB et al. revealed that the commonest psychiatric disorder among psychiatric outpatients is neurotic, stress-related and somatoform disorders (35.4\%) followed by mood disorder (18.3\%), schizophrenia, schizotypal and delusional disorders (17.4\%) and mental and Behavioural disorders due to psychoactive substance use $(9.7 \%)^{12}$. The probable cause of the difference in the prevalence of psychiatric illness in Nepal could be primarily due to the differences in terms of diverse ethnic backgrounds, socio-demographic and socio-economic variations indifferent parts of Nepal.

\section{Socio-Economic, demographic factors and psychiatric disorders:}

In the course of conducting research, most of the Psychiatric disorders were seen among young patients having age groups <40yrs $(n=191)$ and $(n=49)$ patients were $>40 y r s$. Research conducted by Banerjee et al concerning various psychiatric disorders namely schizophrenia, anxiety and depression in Nepal shows that they are commonly witnessed below 40 year ${ }^{13-15}$. Among many common cases of psychiatric disorders, primarily Schizophrenia, Schizotypal and Delusion Disorder patients were $40 \mathrm{yrs}(n=68)$ and $<40 y r s(n=19)$. In terms of Mood (Affective) Disorders, most of the patients were aged<40yrs $(n=48)$. The present study is quite similar to research conducted by Maki $P$ et al as it shows that schizophrenia is common in adolescent and mood disorders which are commonly seen among youths in $\mathrm{Nepal}^{16}$. Psychiatric disorders are commonly seen in young population and the reason behind may be that life other possible reasons may be that patients $>40 \mathrm{yrs}$ are socially more secured and the chances of psychiatric disorders in this population are less ${ }^{17}$. The study indicates that psychiatric disorder is most commonly seen among males as compared to females and this finding resembles similar study carried out in Bangladesh. However, these findings were contradictory to the findings of Australia and the USA as it indicates the dominance of psychiatric illness among females ${ }^{18-20}$. With regard to male patients [ $n=130$ ], Schizophrenia, Schizotypal and Delusion Disorders $43.8 \%$ was common followed by Mood (Affective) Disorders $25.4 \%$. Similar finding was reported by Banerjee et al and it affirmed schizophrenia as common case among males ${ }^{21}$. Among 110 female patients, the majority of them had suffered from Mood (Affective) Disorders 30.9\%, Schizophrenia, Schizotypal and Delusion Disorders $27.3 \%$ followed by Neurotic, stress-related and somatoform disorders $24.5 \%$ respectively. In a study conducted in 2010 among Asians population, it is revealed that Mood (Affective) Disorder like depression is commonly seen among females ${ }^{22}$. The cause of such gender variation on different psychiatric disorders could be due to variances in recurrence risk, emotional expressions, societal roles and professional bias during the time of diagnosis ${ }^{17}$. Unemployment is a serious problem particularly in rural areas in Nepal ${ }^{23}$. In the course of the study, it was found that psychiatric disorders were mainly associated with unemployment problem low monthly income of family. Most of the patients were unemployed $(n=199)$ having monthly income $<10000(n=187)$. Among the unemployed patients $39.2 \%$ were suffering from Schizophrenia, Schizotypal and Delusion Disorders followed by Mood (Affective) Disorders 25.1\% and Neurotic, stress-related and somatoform disorders $13.6 \%$ respectively. Most of the patients had monthly income $<10000$ per month $(n=187)$ and they were suffering from Schizophrenia, Schizotypal and Delusion Disorders ( $n=67)$, mood affective disorders Neurotic, stress-related and somatoform disorders $(n=33)$. Similar finding was noted in a study undertaken among the Poor in United States, England, Japan, Norway, Ireland and Iceland. Unlike this finding, the research conducted in the context of India and Italy showed that the disease is more frequently witnessed among the rich. Schizophrenia was also found to be common among urban dwellers and blackmm community population in the United States ${ }^{24}$. This finding confirms that low socioeconomic status of people is associated with a higher prevalence of psychiatric disorders ${ }^{25,26}$ and similar types of findings were also reported by Risal A in Nepal ${ }^{5}$. Most of the patients were Hindus $(n=200)$ followed by Buddhist ( $n=25)$, Christian ( $n=9)$, and Muslim $(n=6)$. Likewise, from religious point of view, Schizophrenia, Schizotypal and Delusion Disorders was the commonest psychiatric disorder $(n=187)$ among the Hindus followed by mood affective disorders $28.5 \%$, Neurotic, stress-related and somatoform disorders $18.5 \%$ Mental and Behavioural disorder due to Psychoactive Substance abuse 11\% 
respectively. Similar findings was reported by Banerjee et al which has showed that Psychiatric disorders like schizophrenia was commonly found in Hindus followed by Buddhists. This could be primarily owing to reason that Nepal is a Hindu population dominated country and it is expected that the majority of patients will be Hindus ${ }^{21}$. Most of the patient were students $(n=102)$ followed by housewives $(n=74)$ but service holders were very limited in numbers $(n=17)$. Among students, the commonest psychiatric disorder was Schizophrenia, Schizotypal and Delusion Disorders 47.1\%, Mental and Behavioural disorder due to Psychoactive Substance abuse 21.6\%, Mood (Affective) Disorders $19.6 \%$, Neurotic, stress-related and somatoform disorders 7.8\%, Behavioural syndromes associated with physiological disturbances and physical factors $3.9 \%$ respectively. None of the students were diagnosed to be suffering from Organic including symptomatic mental disorder $0 \%$ or mental retardation $0 \%$. However, among housewives, the commonest psychiatric disorder was Mood (Affective) Disorders and Schizophrenia, Schizotypal and Delusion Disorders 31\% whereas none of the housewives were found to be suffering from Mental retardation 0\%.Similar finding was reported by Fahmida $A$ et al in Bangladesh and it revealed that the occupation related Psychiatric disorders were rather common among housewives and students ${ }^{18}$. Study done by Banerjee et al in Nepal also showed that psychiatric disorders such as mood affective disorders; Neurotic, stress-related and somatoform disorders are commonly noted among housewives and students. The most convincing reason behind this fact is stressful life pattern and the effects resulted due to child birth which may lead to various psychiatric disorders among housewives ${ }^{18}$. Other social problems such as broken families, living apart from husband or husband residing in foreign country for employment purpose can also be attributed to this fact ${ }^{13}$. However, among students, some causes of high prevalence psychiatric disorders could be due to stressful life and inability to cope up with their studies ${ }^{14}$. This study which was conducted from ethnic line of patients indicated that Psychiatric disorder was commonly found among Dalits [ $n=72]$ followed by Brahmin [ $n=66$ ], Chettri [ $n=46]$, Newar [ $=$ 19], Gurung [ $n=17]$, others [ $=13$ ] and Magar and Pun [ $=$ 7] respectively. There are similar other findings which report that psychiatric disorders are commonly found in Dalit in Nepal ${ }^{1}$. Among the Dalit, the commonest psychatric disorders were Schizophrenia, Schizotypal and Delusion Disorders $30.6 \%$ followed by Mental and behavioral disorder due to Psychoactive Substance abuse $20.8 \%$, mood and affective disorders $18.1 \%$, Neurotic, stress-related and somatoform disorders $12.5 \%$. Study done by Banerjee et al also reported that schizophrenia is commonly found among Dalit patients. Similar findings were reported in the research conducted in the Rural Community of Nepal as well ${ }^{1,17}$. It is because Nepal is an under developed country having poor financial status which could lead to high prevalence of psychiatric illness. Nepal is a mountainous country and her main source of income is agriculture. Many Nepalese people are financially poor and therefore can't afford to have health care facility timely and in course of time it may result in various psychiatric disorders. A research conducted in Nepal by Kohrt BA et al also concluded that the caste-based disparity, poverty, lack of social support and stressful life events have adversely affected the mental health of Nepalese people ${ }^{1}$.

\section{Conclusion}

Psychiatric disorders namely Schizophrenia was the commonest psychiatric disorder among the low socioeconomic group among the culture Dalit. In Nepal it is also found that culture based differences in mental health in mediated by poverty, unemployment, dearth of family income could lead to high prevalence of psychiatric illness among the Nepalese population. Depending on these finding of the study interventions should target these factors to decline the load of various psychiatric illness among the Nepalese population

\section{Limitation of the study}

This research is based on the hospital study from Western Development Region of Nepal. A multi centric hospital based study with higher sample size will be beneficial to assess the psychiatric disorders in different cultures all over Nepal.

\section{Relevance of the study:}

Psychiatric disorders namely Schizophrenia was the commonest psychiatric disorder among low socio-economic group of people who belong to Dalit community. It is also revealed that culture based differences have worsened the quality of mental health and moreover it has been further complicated by poverty, unemployment, dearth of family income leading to high prevalence of psychiatric illness among Nepalese population. Based on these findings, interventions should target to address these factors to minimize the load of various psychiatric illnesses.

\section{Future scope of the study:}

This study is exclusively based on the research which was carried out in the territory hospital located in Western Development Region in Nepal. A multi centric hospital based research having bigger sample size would be beneficial to assess the psychiatric disorders in the light of different ethnic and cultural backgrounds of Nepalese people.

\section{Author's Contribution:}

IB designed the study, deduced the data, drafted the manuscript, and revised it. IB2, BR, PKC and SK planned the study with IB, acquired the data, conducted the data analysis, interpreted the data, and revised the manuscript. IB2 has also participated in the language editing along with IB and SK. BS participated in statistical analysis, interpreted the data, and revised the manuscript. PKS, PB and ACS critically revised the manuscript. All the authors approved the final document. 
List of abbreviations

NPR: Nepalese rupees Yrs: Years MTH: Manipal teaching hospital.

\section{Acknowledgements:}

We prolong our deepest and warm gratitude to Late $\mathrm{Dr}$ Akhilesh Chandra Jauhari, Professor, Department of Pharmacology, Manipal College of Medical sciences, Nepal for all guidance, proposition and cooperation in writing this paper. We are grateful to Dr. B. M. Nagpal, Dean and CEO, MCOMS, Nepal. We are grateful to $\mathrm{Dr}$ S. M. Banerjee, Orthopaedic surgeon, Kalyani, West Bengal, India and $\mathrm{K}$ Ramesh, HOD, Psychiatry for constant help and support.

\section{Conflict of interest:}

There is no conflict of interest among authors arising from the study.

\section{References:}

1. Kohrt BA, Speckman RA, Kunz RD, Baldwin JL, Upadhaya N, Acharya NR, Sharma VD, Nepal MK, Worthman CM. Culture in psychiatric epidemiology: using ethnography and multiple mediator models to assess the relationship of caste with depression and anxiety in Nepal. Ann Hum Biol. 2009 May-Jun;36(3):261-80.

http://dx.doi.org/10.1080/03014460902839194 PMid:19381985 PMCid:PMC3907946

2. Aryal UR, Vaidya A, Shakya-Vaidya S, Petzold M, Krettek A. Establishing a health demographic surveillance site in Bhaktapur district, Nepal: initial experiences and findings. BMC Res Notes. 2012 Sep 5;5:489. http://dx.doi.org/10.1186/1756-0500-5-489

PMid:22950751 PMCid:PMC3494612

3. Jha A, Ranjan S, Pradhan PK, Jha T. Challenges of setting up psychiatric services in Nepal: Lessons from the first year of Janakpur Project. Asian J Psychiatr. 2011 Dec;4(4):297-9.

http://dx.doi.org/10.1016/j.ajp.2011.10.004

PMid:23051166

4. Shrestha MR, Sherchan S, Shakya R, Joshi D. Monthly pattern of psychiatric morbidity and duration of stay among the patients admitted in Mental Hospital, a central level tertiary care hospital. Nepal Med Coll J. 2011 Jun;13(2):133-6.

PMid:22364100

5. Risal A. Common mental disorders. Kathmandu Univ Med J (KUMJ). 2011 Jul-Sep;9(35):213-7.

PMID: 22946143

6. Shankar PR, Partha P, Shenoy N: Self-medication and non-doctor prescription practices in Pokhara valley. Western Nepal: a questionnaire based study. BMC Fam Pract. 2002; Sep 17, 3:17. http://dx.doi.org/10.1186/1471-2296-3-17 PMid:12236905 PMCid:PMC130019

7. World Health Organization: The ICD-10 Classification of mental and Behavioural disorders (Tenth Revision). Geneva: Diagnostic criteria for research; 1992.
8. Sathian B, Sreedharan J, Baboo NS, Sharan K, Abhilash ES, Rajesh E. Relevance of Sample Size Determination in Medical Research. Nepal Journal of Epidemiology 2010; $1(1): 4-10$.

http://dx.doi.org/10.3126/nje.v1i1.4100

9. Sathian B: Reporting dichotomous data using Logistic Regression in Medical Research: The scenario in developing countries. Nepal Journal of Epidemiology 2011; 1(4):111-113.

http://dx.doi.org/10.3126/nje.v1i4.5752

10. Risal A, Sharma PP. Psychiatric morbidity patterns in referred inpatients of other specialties. Nepal Med Assoc. 2013; Jan-Mar;52(189):238-44.

PMID: 23591303

11. Tausig M, Subedi S, Subedi J, Ross J, Broughton LC, Singh R, Blangero J, Williams-Blangero S: Mental illness in Jiri Nepal. The Jirel issue Jan 2000, 27:105-15.

12. Khattri JB, Godar ST, Thapa P, Ramesh K, Chakrabortty PK, Thapa BB. Socio-demographic characteristics and diagnostic profile of patients attending psychiatric OPD of a private hospital in western region of Nepal. Nepal Journal of Medical Sciences. 2012; 1(1): 15-18. http://dx.doi.org/10.3126/njms.v1i1.5790

13. Banerjee I, Roy B, Sathian B, Banerjee I, Kumar SS, Saha A: Medications for Anxiety: A Drug utilization study in Psychiatry Inpatients from a Tertiary Care Centre of Western Nepal. Nepal Journal of Epidemiology 2010, 1(4):119-25.

http://dx.doi.org/10.3126/nje.v1i4.5753

14. Banerjee I, Roy B, Banerjee I, Sathian B, Mondol M, Saha A: Depression and its Cure: A Drug Utilization Study from a Tertiary Care Centre of Western Nepal. Nepal Journal of Epidemiology 2011, 1(5):144-52. http://dx.doi.org/10.3126/nje.v1i5.6152

15. Banerjee I, Roy B, Sathian B, Banerjee I, Chakraborty PK, Saha A. Socio demographic profile and utilization pattern of antipsychotic drugs among schizophrenic inpatients: a cross sectional study from western region of Nepal. BMC Psychiatry. 2013 Mar 22;13:96. http://dx.doi.org/10.1186/1471-244X-13-96

PMid:23522357

16. Maki P, Veijola J, Jones PB, Murray GK, Koponen $H$, Tienari $P$, Miettunen J, Tanskanen $P$, Wahlberg KE, Koskinen J, Lauronen $E$, Isohanni $M$ : Predictors of schizophrenia-a review. Br Med Bull 2005 Jun 9, 73-74:115.

http://dx.doi.org/10.1093/bmb/ldh046

PMid:15947217

17. Khattri JB, Poudel BM, Thapa P, Godar ST, Tirkey S, Ramesh K, Chakrabortty PK. An Epidemiological Study of Psychiatric Cases in a Rural Community of Nepal. Nepal Journal of Medical Sciences 2013;2(1):52-6. http://dx.doi.org/10.3126/njms.v2i1.7654

18. Fahmida A, Wahab MA, Rahman MM: Pattern of psychiatric morbidity among the patients admitted in a 
private psychiatric clinic. Bangladesh Journal of Medical Science 2009, 8(1-2):23-28.

19. Mant A, Lansbury G, Bridges-Webb C: Trends in psychotropic drug prescribing in Australia. Med J Aust 1987,146(4):208-10.

PMid:3574214

20. Mendelson T, Kubzansky LD, Datta GD, BukaSL.Relation of female gender and low socioeconomic status to internalizing symptoms among adolescents: a case of double jeopardy? SocSci Med. 2008 Mar;66(6):1284-96. http://dx.doi.org/10.1016/j.socscimed.2007.11.033

PMid:18248868

21. Hector M. Gonzalez, WassimTarraf, Brady T. West, Domin Chan, Patricia Y. Miranda and Fredrick T. Leong. Antidepressant Use among Asians in the United States.Depression and Anxiety. 2010; 27(1): 46-55.

http://dx.doi.org/10.1002/da.20636

PMid:20013960 PMCid:PMC2805045

22. Pradhanang AL.Demographic situation and development in Nepal. Econ J Nepal. 1983 Oct-Dec;6(4):11-22.

PMid:12339820

23. Foster HD: The Geography of Schizophrenia: Possible Links with Selenium and Calcium Deficiencies, Inadequate Exposure to Sunlight and Industrialization. Journal of Orthomolecular Medicine 1988, 3(3):135-40.

24. Mackenbach JP, Stirbu I, Roskam A-JR, Schaap MM, Menvielle $G$, Leinsalu $M$, Kunst $A E$, the European Union Working Group on Socioeconomic Inequalities in $\mathrm{H}$ : Socioeconomic inequalities in health in 22 European countries.NEngl J Med 2008, 358(23):2468-2481.

http://dx.doi.org/10.1056/NEJMsa0707519

PMid:18525043

25. Lorant V, Deliege D, Eaton W, Robert A, Philippot P, Ansseau $M$ : Socioeconomic inequalities in depression: a meta-analysis.Am J Epidemiol 2003, 157(2):98-112.

http://dx.doi.org/10.1093/aje/kwf182

PMid:12522017

26. Lesen $E$, Anderrson $K$, Petzoid $M$, Carlsten $A$ : Socioeconomic determinants of Psychotropic drug utilization among elderly: a national population based cross sectional study. BMC Publ Health 2010, 10:118.

http://dx.doi.org/10.1186/1471-2458-10-118

PMid:20214796 PMCid:PMC2845562

\begin{tabular}{|l|l|}
\hline \multicolumn{2}{|c|}{ Article Information } \\
\hline \multicolumn{2}{|c|}{ Article history } \\
\hline Received & $5^{\text {th }}$ December 2013 \\
\hline Received in revised form & $12^{\text {nd }}$ March2013 \\
\hline Accepted & $20^{\text {th }}$ March 2014 \\
\hline
\end{tabular}

